\title{
Phase transition, spin-charge separation, and spin filtering in a quantum dot
}

\author{
Michael Pustilnik ${ }^{1}$ and László Borda ${ }^{2}$ \\ ${ }^{1}$ School of Physics, Georgia Institute of Technology, Atlanta, GA 30332 \\ ${ }^{2}$ Research Group "Theory of Condensed Matter" of the Hungarian Academy of Sciences, \\ Institute of Physics, TU Budapest, Budafoki út 8., H-1521, Hungary
}

\begin{abstract}
We consider low temperature transport through a lateral quantum dot asymmetrically coupled to two conducting leads, and tuned to the mixed-valence region separating two adjacent Coulomb blockade valleys with spin $S=1 / 2$ and $S=1$ on the dot. We demonstrate that this system exhibits a quantum phase transition driven by the gate voltage. In the vicinity of the transition the spin on the dot is quantized, even though the fluctuations of charge are strong. The spin-charge separation leads to an unusual Fano-like dependence of the conductance on the gate voltage and to an almost perfect spin polarization of the current through the dot in the presence of a magnetic field.

PACS numbers: 72.15.Qm, 73.23.Hk, 73.63.Kv
\end{abstract}

In a single-electron transistor setup [1] the number of electrons $N$ in a quantum dot is controlled by the potential on the capacitively coupled gate electrode. At low temperature $N$ is close to an integer at almost any gate voltage, except narrow mixed-valence regions, where adding a single electron to the dot does not lead to a large penalty in electrostatic energy. The distance between these regions sets the scale for the dependence of measurable quantities on the gate voltage, which makes it convenient to use a dimensionless parameter $N_{0}$, the gate voltage normalized by this scale. In terms of $N_{0}$, the mixed-valence regions are narrow intervals of the width

$$
\Delta_{N} \sim \Gamma / E_{C} \ll 1,
$$

about half-integer values of $N_{0}[2]$. Here $E_{C}$ is the charging energy and $\Gamma$ is the tunneling-induced width of singleparticle energy levels in the dot.

In a typical experiment a dot is connected via tunneling junctions to two massive electrodes [1]. At temperatures in the range $\Gamma \lesssim T \ll E_{C}$, the conductance $G$ is suppressed outside the mixed-valence regions, resulting in a quasiperiodic sequence of well-defined Coulomb blockade peaks in the dependence $G\left(N_{0}\right)$ [1, 2]. When $T$ is further lowered, $G\left(N_{0}\right)$ changes dramatically due to the onset of the Kondo effect [2, 3]. At $T \rightarrow 0$ pairs of adjacent Coulomb blockade peaks merge to form broad maxima at $N \approx$ odd integer, separated by smooth crossovers from the minima at $N \approx$ even integer. Although $G\left(N_{0}\right)$ at $T \gtrsim \Gamma$ is very different from that at $T \ll \Gamma$, in the mixedvalence regions both functions are featureless [2, 3].

The evolution of $G$ towards its low-temperature limit can be rather complicated. Indeed, GaAs quantum dots with odd $N$ usually have spin $S=1 / 2$ [4, 5]. In this case the dependence $G(T)$ is characterized by a single energy scale, the Kondo temperature $T_{K} ; G(T)$ increases monotonically with the decrease of $T$ at $T \ll \Gamma[2]$. However, dots with even $N$ often have spin $S=1$ rather than zero [4, 5, 6]. Kondo effect then occurs in two stages, controlled by two different energy scales, $T_{K}$ and $T_{K}^{\prime}<T_{K}$ [7]. The resulting $G(T)$ is not monotonic: $G$ first raises, and then drops again when $T$ is lowered [7, 8]. The dependence of the conductance on the Zeeman energy $B$ of an applied magnetic field is also non-monotonic and is characterized by the same scales $T_{K}$ and $T_{K}^{\prime}[$ ].

The values of $T_{K}$ and $T_{K}^{\prime}$ depend on $N_{0}$ and their ratio, in general, is not tunable. A notable exception occurs when the conductances of the dot-lead junctions are very different, i.e. when the width $\Gamma=\Gamma_{L}+\Gamma_{R}$ is dominated by the contribution from the lead with the stronger coupling to the dot, say, $\Gamma_{L} \gg \Gamma_{R}$. (Note that conductances of the junctions are easily tunable in lateral quantum dot systems such as those studied in [8]). It can be shown 2] that in this limit $T_{K}^{\prime} \ll T_{K}$ for all $N_{0}$. In particular, in the vicinity of the mixed-valence region $T_{K}^{\prime} \sim \Gamma_{R}$ while $T_{K} \sim \Gamma_{L} \gg T_{K}^{\prime}$. Accordingly, the second stage of the Kondo effect will not develop if

$$
\Gamma_{R} \ll \max \{T, B\} \ll \Gamma_{L} .
$$

In this paper we show that under these conditions the conductance in the mixed-valence region between the Coulomb blockade valleys with $S=1 / 2$ and $S=1$ on the dot varies with $N_{0}$ on the scale which is parametrically small compared with $\Delta_{N}$, in striking difference with the conventional smooth dependence described above.

The dependence of the conductance on $B$ at $B \gg T$ is qualitatively similar to its dependence on $T$ at $T \gg B$ [2]. Since $B$-dependence is much easier to understand, we concentrate here on the limit $T \rightarrow 0$ (the effect of a finite $T$ is briefly discussed towards the end of the paper).

The first inequality in Eq. (2) allows one to take into account the coupling to the right lead in the lowest nonvanishing order in $\Gamma_{R} / \Gamma_{L}$. The conductance at any finite $B$ is then given by [2]

$$
g=g_{\uparrow}+g_{\downarrow}, \quad g_{s}=\sin ^{2} \delta_{s} .
$$

Here $g=G / G_{0}$ is the conductance normalized by $G_{0} \sim$ $\left(e^{2} / h\right) \Gamma_{R} / \Gamma_{L}$, the largest value conductance per spin can reach for strongly asymmetric coupling to the leads; $g_{s}$ is the conductance (in units of $G_{0}$ ) for electrons with spin 
$s$, and $\delta_{s}$ is scattering phase shift at the Fermi level for electrons with spin $s$ in the left lead.

In order to calculate the phase shifts, we model a quantum dot coupled to a single lead by the Hamiltonian

$$
H=H_{0}+H_{t}+H_{d}
$$

The first term here describes the electrons in the lead. For a lateral quantum dot it is sufficient to take into account only a single propagating mode [2, 7],

$$
H_{0}=\sum_{k s} \xi_{k} \psi_{k s}^{\dagger} \psi_{k s}
$$

and the spectra $\xi_{k}$ can be linearized near the Fermi level, which corresponds to a constant density of states $\nu$.

The second term in Eq. (4) describes the tunneling coupling between the dot and the lead,

$$
H_{t}=\sum_{n k s} t_{n} \psi_{k s}^{\dagger} d_{n s}+\text { H.c. }
$$

In the following we set $t_{n}=t$, so that all levels in the dot have the same width $\Gamma=\pi \nu t^{2}$. This assumption is not essential for the validity of the following consideration.

The last term in Eq. (4) describes an isolated dot,

$$
H_{d}=\sum_{n s} \epsilon_{n} d_{n s}^{\dagger} d_{n s}+E_{C}\left(\hat{N}-N_{0}\right)^{2}-E_{S} \hat{\mathbf{S}}^{2}-B \hat{S}_{z}
$$

Here $\hat{N}=\sum_{n s} d_{n s}^{\dagger} d_{n s}$ and $\hat{\mathbf{S}}=\frac{1}{2} \sum_{n s s^{\prime}} d_{n s}^{\dagger} \hat{\boldsymbol{\sigma}}_{s s^{\prime}} d_{n s^{\prime}}$ are operators of the total number of electrons on the dot, and of the dot's spin, respectively $\left(\hat{\boldsymbol{\sigma}}=\left(\hat{\sigma}_{x}, \hat{\sigma}_{y}, \hat{\sigma}_{z}\right)\right.$ are Pauli matrices). For a typical dot the parameters $\delta E$ (mean single-particle level spacing), $E_{S}$ (exchange energy), and $E_{C}$ (charging energy) satisfy $E_{S} \ll \delta E \ll E_{C}$ [2].

An isolated dot with even $N$ will have $S=1$ in the ground state if the spacing $\varepsilon$ between the two singleparticle levels closest to the Fermi level is anomalously small, $2 E_{S}-\varepsilon>0$ [4, 5, 6]. For simplicity, we assume here that $2 E_{S}-\varepsilon \gtrsim \Gamma$. Although this simplification imposes a stronger restriction on $\Gamma$ then $\Gamma \ll \delta E$ (this inequality justifies the tunneling Hamiltonian description of the dot-lead junction [2]), it does not affect the results.

For the model (4)-(7) the phase shifts are given by

$$
\delta_{\uparrow}=(\pi / 2)(N+M), \quad \delta_{\downarrow}=(\pi / 2)(N-M),
$$

where $N=\langle\hat{N}\rangle$ is the number of electrons in the dot, and $M=2\left\langle\hat{S}_{z}\right\rangle$ is the dot's magnetization [9]. We start with $N_{0}$ outside the mixed-valence region,

$$
\Delta_{N} \ll\left|N_{0}-\tilde{N}_{0}\right| \ll 1, \quad \widetilde{N}_{0}-1 / 2=\text { odd integer }
$$

Here $N \approx \widetilde{N}_{0} \pm 1 / 2$ is close to an integer. The tunnelinginduced virtual transitions to states with "wrong" $N$ can be "integrated out" with the help of the Schrieffer-Wolff transformation, yielding an effective Kondo Hamiltonian

$$
H=H_{0}+V \rho+J(\mathbf{s} \cdot \mathbf{S})-B S_{z},
$$

where $\rho=\sum_{k k^{\prime} s} \psi_{k s}^{\dagger} \psi_{k^{\prime} s}$ and $\mathbf{s}=\frac{1}{2} \sum_{k k^{\prime} s s^{\prime}} \psi_{k s}^{\dagger} \hat{\boldsymbol{\sigma}}_{s s^{\prime}} \psi_{k^{\prime} s^{\prime}}$ are operators describing the local particle and spin densities of conduction electrons. The operator $\mathbf{S}$ in Eq. (10) is a projection of $\hat{\mathbf{S}}$ [see Eq. (7)] on the ground state multiplet of an isolated dot with fixed integer $N$. The reduction of the microscopic model (4)-(7) to the Kondo Hamiltonian (10) is valid only when $N_{0}$ is outside the mixed-valence region and at sufficiently low energies,

$$
\left|\xi_{k}\right| \lesssim D=\min \left\{d_{N}, 2 E_{C}\left|N_{0}-\tilde{N}_{0}\right|\right\}
$$

where $d_{N}=\delta E\left(d_{N}=2 E_{C}-\varepsilon\right)$ for odd (even) $N$. The parameters $V$ and $J$ in Eq. (10) can be estimated as [2, 7]

$$
\nu V \sim \Delta_{N}\left(N_{0}-\tilde{N}_{0}\right)^{-1}, \quad \nu J \sim|\nu V| .
$$

(It should be noted that $V$ and $J$ are subject to strong mesoscopic fluctuations; the order-of-magnitude estimate (12) is sufficient for our purpose).

The potential scattering term in Eq. (10) is responsible for the deviations $\delta N$ of the dot's occupation from the corresponding integer values $\widetilde{N}_{0} \pm 1 / 2$,

$$
\delta N=N-\left(\tilde{N}_{0} \pm 1 / 2\right) \approx-2 \nu V \sim \Delta_{N}\left(\tilde{N}_{0}-N_{0}\right)^{-1}
$$

Note that $|\delta N|$ is finite and increases with approach to the mixed-valence region. Also note that a weak magnetic field $B \ll \Gamma$ does not affect $N\left(N_{0}\right)$.

On the contrary, $M\left(N_{0}\right)$ depends strongly on $B$. Indeed, $M(B)$ for a given $N_{0}$ is controlled by the Kondo temperature $T_{K}\left(N_{0}\right)$, which can be estimated from

$$
\ln \left(D / T_{K}\right) \sim(\nu J)^{-1} \sim \Delta_{N}^{-1}\left|N_{0}-\widetilde{N}_{0}\right|
$$

Accordingly, $T_{K} \sim \Gamma$ at $\left|N_{0}-\widetilde{N}_{0}\right| \sim \Delta_{N}$, and decreases exponentially with the increase of the distance to $\tilde{N}_{0}$. A fixed field $B$ is large compared to $T_{K}$ at $\left|N_{0}-\widetilde{N}_{0}\right| \gg \Delta_{B}$, where $\Delta_{B}$ is the distance between $N_{0}$ and $\tilde{N}_{0}$ at which $B \sim T_{K}\left(N_{0}\right)$. In this regime

$$
M / M_{0}=1-\left[2 \ln \left(B / T_{K}\right)\right]^{-1},
$$

where $M_{0}=1(2)$ for $N_{0}<\widetilde{N}_{0}\left(N_{0}>\widetilde{N}_{0}\right)$. In the opposite limit $\Delta_{B} \gg\left|N_{0}-\widetilde{N}_{0}\right| \gg \Delta_{N}$ (note that $\Delta_{B} \gg \Delta_{N}$ for $B \ll \Gamma)$ the system is in the strong coupling regime $B \ll T_{K}$. Here $M\left(N_{0}\right)$ depends strongly on the parity of $N$. Indeed, $\mathbf{S}$ in Eq. (10) is spin-1/2 operator for odd $N$ (i.e. for $\widetilde{N}_{0}-N_{0} \gg \Delta_{N}$ ), and spin-1 operator for even $N\left(N_{0}-\tilde{N}_{0} \gg \Delta_{N}\right)$. This difference is crucial. An antiferromagnetic local exchange interaction with a 
single species of itinerant electrons suffices to completely screen $S=1 / 2$ magnetic impurity, thereby forming a singlet (non-degenerate) ground state 10, 11, 12, 13. In this case the approach to the low-energy fixed point is Fermi-liquid-like [1], and

$$
M \sim B / T_{K}
$$

On the contrary, for $S=1$ only half of the impurity's spin is screened, and the ground state is a doublet 11, 12. The low-energy physics is then described by the ferromagnetic exchange of the conduction electrons with the remaining spin $S=1 / 2$ [11, 12], and

$$
M=1+\left[2 \ln \left(T_{K} / B\right)\right]^{-1}
$$

Although the above results were obtained for $N_{0}$ outside the mixed-valence region $\left|N_{0}-\widetilde{N}_{0}\right| \lesssim \Delta_{N}$, some conclusions regarding this region can be drown as well. Indeed, since $\delta N$ in Eq. (13) is finite, it is plausible that $N$ varies continuously with $N_{0}$, as sketched in Fig. प(a). (a)

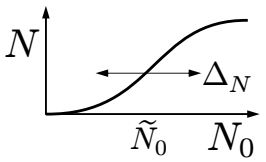

(c)

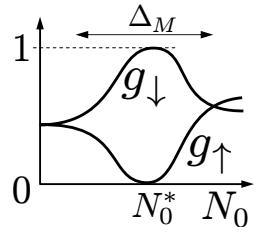

(b)

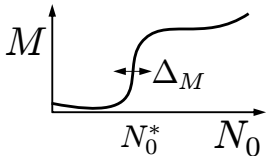

(d)

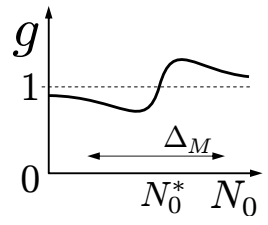

FIG. 1: (a) Number of electrons in the $\operatorname{dot} N$ differs appreciably from an integer in a narrow mixed-valence region of the width $\Delta_{N}$. (b) At $B \ll \Gamma$, the width $\Delta_{M}$ of the crossover region in the dependence of the magnetization $M$ on the gate voltage $N_{0}$ is small compared to $\Delta_{N}$. (c) Spin-resolved conductances in the crossover region at $T \ll B \ll \Gamma$. (d) The total conductance at $\max \{B, T\} \ll \Gamma$.

The dependence $M\left(N_{0}\right)$ is more complicated. Consider the limit $B \rightarrow+0$. In this limit $M$ is determined solely by the ground state degeneracy. Since the degeneracy can not change continuously, the system must go through a quantum phase transition (QPT) at a certain value of $N_{0}=N_{0}^{*}$. As shown above, the ground state is either a singlet or a doublet when the charge fluctuations are weak. Therefore, the transition must occur within the mixed-valence region, i.e. $\left|N_{0}^{*}-\widetilde{N}_{0}\right| \lesssim \Delta_{N}$. The QPT manifests itself in a singular dependence of the magnetization $M$ on the gate voltage,

$$
\lim _{B \rightarrow+0} M=\theta\left(N_{0}-N_{0}^{*}\right) .
$$

Note that in the vicinity of the transition the spin is quantized, even though the fluctuations of charge are very strong, $N^{*}=N\left(N_{0}^{*}\right) \approx$ half-integer (unlike in the case of transitions that occur at a fixed integer $N$ 14, 15]).

Any finite field lifts the degeneracy of the ground state. QPT then turns to a crossover, and the sharp step in the dependence of $M\left(N_{0}\right)$ is smeared. The crossover takes place in a narrow interval of gate voltages $\left|N_{0}-N_{0}^{*}\right| \lesssim \Delta_{M}$. We expect that at a sufficiently low field the crossover width $\Delta_{M}$ remains to be small compared to $\Delta_{N}$, see Fig. 1(b). In order to estimate $\Delta_{M}$, we now construct an effective Hamiltonian $H_{\mathrm{QPT}}$ for the vicinity of the transition.

Such Hamiltonian should be applicable at low energies $(B, T \ll \Gamma)$ and for $N_{0}$ in the range $\left|N_{0}-N_{0}^{*}\right| \ll \Delta_{N}$, which includes the crossover region. At these energies and gate voltages the number of electrons in the dot is approximately constant, $N \approx N^{*}$, while $M\left(N_{0}\right)$ changes rapidly. It is therefore plausible that $H_{\mathrm{QPT}}$ acts only on the spin degrees of freedom (spin-charge separation). At energies below $\Gamma$ half of the dot's spin when it is in the triplet state is already screened. The simplest possible model accounting for the interaction of the (still unscreened) spin- $1 / 2$ with electrons in the narrow strip of energies $\left|\xi_{k}\right| \lesssim \Gamma$ reads

$$
H_{\mathrm{QPT}}=H_{0}^{\prime}+J^{\prime}\left(\mathbf{s}^{\prime} \cdot \mathbf{S}\right)-B S^{z} .
$$

Here $H_{0}^{\prime}$ and $\mathbf{s}^{\prime}$ [cf. $H_{0}$ and $\mathbf{s}$ in Eq. (10)] are defined in terms of the operators $\psi_{k s}^{\prime}$ acting in the basis of singleparticle states that incorporate an extra scattering phase shift $\delta^{*}=\pi N^{*} / 2$.

For $H_{\mathrm{QPT}}$ to describe the change of the ground state symmetry at $N_{0}=N_{0}^{*}$, the exchange constant $J^{\prime}$ must change its sign at this point 10, 11, 12]. Assuming the dependence $J^{\prime}\left(N_{0}\right)$ to be analytical, we can write

$$
\nu J^{\prime}\left(N_{0}\right) \sim \Delta_{N}^{-1}\left(N_{0}^{*}-N_{0}\right) .
$$

The coefficient in Eq. (20) has been chosen in such a way that $\nu J^{\prime} \sim 1$ for $N_{0}^{*}-N_{0} \sim \Delta_{N}$. This ensures the continuity of $M\left(N_{0}\right)$ throughout the singlet side of the transition $N_{0}<N_{0}^{*}$.

A comment on the status of Eqs. (19), (20) is in order here. The effective low-energy Hamiltonian $H_{\mathrm{QPT}}$ is in the same relation to the original microscopic model (4)-(17) as, e.g., the effective Fermi-liquid description of strong coupling regime [11] is to the Kondo model. As in the latter case, the applicability of $H_{\mathrm{QPT}}$ can be verified by comparing the predictions of the two models.

The magnetization for the model (19), (20) is obtained using the standard scaling arguments [10]. Very close to the transition (when $\left|\nu J^{\prime}\right| \ln (\Gamma / B) \ll 1$ ) the first order perturbation theory in $\nu J^{\prime} \ll 1$ yields

$$
M-1 \approx-\nu J^{\prime} / 2 \sim \Delta_{N}^{-1}\left(N_{0}-N_{0}^{*}\right) .
$$

On the doublet side $M\left(N_{0}\right)$ slowly increases with the distance to the transition, saturating at

$$
M=1+[2 \ln (\Gamma / B)]^{-1} .
$$


Note that Eq. (22) matches Eq. (17) at the border of the mixed-valence region.

On the singlet side of the transition $\left(N_{0}<N_{0}^{*}\right)$ the magnetization is given by Eq. (15) (with $M_{0}=1$ ) for $B \gg T_{K}$ and by Eq. (16) for $B \ll T_{K}$, where the Kondo temperature $T_{K}\left(N_{0}\right)$ satisfies

$$
\ln \left(\Gamma / T_{K}\right)=\left(\nu J^{\prime}\right)^{-1} \sim \Delta_{N}\left(N_{0}^{*}-N_{0}\right)^{-1} .
$$

$T_{K}$ increases with the distance to the QPT from $T_{K}=0$ at $N_{0}=N_{0}^{*}$ to $T_{K} \sim \Gamma$ at the border of the mixed valence region, where it matches Eq. (14).

As $N_{0}$ is tuned through the mixed-valence region $M$ grows monotonically from $M \sim B / \Gamma \ll 1$ to the value given by Eq. (22). The increase takes place mainly in a narrow interval on the singlet side of the transition where $B \lesssim T_{K}\left(N_{0}\right)$. Eq. (23) then yields the estimate

$$
\Delta_{M} \sim \frac{\Delta_{N}}{\ln (\Gamma / B)} .
$$

The evolution of the phase shifts with $N_{0}$ can now be deduced from Eq. (8). To the left of the crossover [see Fig. [1(b)] both phase shifts are given by $\delta_{s} \approx \pi N^{*} / 2$ with $N^{*} \approx \widetilde{N}_{0}$, see Eq. (9). As $N_{0}$ is tuned through the crossover, $\delta_{\uparrow}$ raises, while $\delta_{\downarrow}$ drops by approximately $\pi / 2$. Therefore the phase shifts necessarily pass through, respectively, the anti-resonance $\delta_{\uparrow}=0(\bmod \pi)$ and resonance $\delta_{\downarrow}=\pi / 2(\bmod \pi)$. Hence, within the crossover region the conductances (3) satisfy $g_{\uparrow} / g_{\downarrow} \ll 1$, and $g_{\uparrow}$ vanishes identically at some value of $N_{0}$. In other words, the system acts as a perfect spin filter.

Details of the dependencies $g_{s}\left(N_{0}\right)$ are sensitive to the dot's occupation at the transition $N^{*}$. While $N^{*}$ is close to a half-integer, it's precise value is obviously non-universal. For example, $N^{*}$ depends on the values of $t_{n}$ for all $n$ in Eq. (6). In Fig. 11(c) we sketch $g_{s}\left(N_{0}\right)$ for $0<\alpha \ll 1$, where $\alpha=N^{*}-\widetilde{N}_{0}$. The dependence of the total conductance $g$ on $N_{0}$ in this case has a characteristic Fano-like shape, see Fig. [1(d).

In order to verify the applicability of the effective Hamiltonian (19), we performed extensive numerical renormalization group (NRG) 13] simulations. For this purpose, we truncated the dot's Hamiltonian (7) to that of a two-level system [15] with

$$
\epsilon_{n}=n \varepsilon / 2, \quad n= \pm 1 .
$$

The NRG data, see Fig. 2] are indeed in an excellent agreement with the behavior expected from Eq. (19). The sharpening of the step in the dependence $M\left(N_{0}\right)$ with the decrease of $B$, obvious in Fig. 22(a), is described very well by $\Delta_{M} / \Delta_{N}=a\left[\ln ^{-1}(\Gamma / B)+b \ln ^{-2}(\Gamma / B)\right]$ with $a=3.0$ and $b=9.5$; at low field this agrees with Eq. (24). Here we defined $\Delta_{M}$ as the distance in $N_{0}$ between the points where $M=0.5$ and 1 , and $\Delta_{N}$ as the distance between the points in Fig. 2(b) where $N=1.25$ and 1.75 .

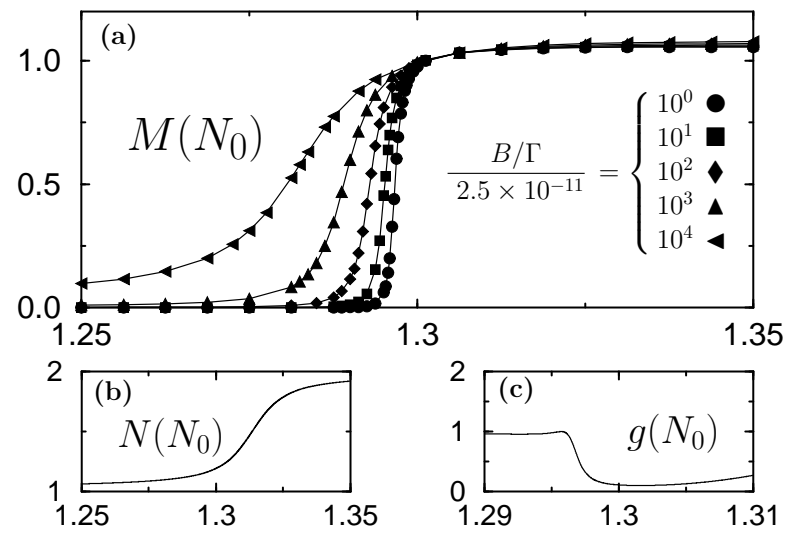

FIG. 2: Results of NRG simulations of the model (4)-(7), (25) with $\Gamma / E_{C}=0.05, E_{S} / E_{C}=0.16$, and $\varepsilon / E_{C}=0.1$.

(a) Magnetization $M\left(N_{0}\right)$ at different $B$. At the transition $M=1$ independently of $B$, in agreement with Eq. (21). (b) Dot's occupation $N\left(N_{0}\right)$. (c) Conductance $g\left(N_{0}\right)$ at $B / \Gamma=$ $2.5 \times 10^{-11}$. Note that in this case $\alpha=N^{*}-3 / 2 \approx-0.25<0$, hence the difference with Fig. 11(d).

So far, we considered the conductance at $T=0$. The above results are valid as long as $T \ll B$; corrections to $g_{s}$ in this case are of the order of $(T / B)^{2}$, and the spin-filtering property remains intact: $\min \left\{g_{\uparrow} / g_{\downarrow}\right\} \sim$ $(T / B)^{2} \ll 1$. At $T \gg B$ the field has a negligible effect. The dependence $g\left(N_{0}\right)$ in this limit is very similar to that at $B \gg T$, see Fig. 1(d) and 2(c), with $T$ replacing $B$ in the crossover width Eq. (24). This peculiar dependence will be observable already at moderately low temperatures $T \lesssim \Gamma$ (note that the observability of the conventional Kondo effect requires $T \lesssim \min \left\{T_{K}\right\} \ll \Gamma$ ).

To conclude, we studied a lateral quantum dot asymmetrically coupled to two conducting leads, and tuned to the mixed-valence region between the Coulomb blockade valleys with $S=1 / 2$ and $S=1$ on the dot. This regime can be realized in devices such as those studied in [8]. We predict that, contrary to naive expectations, the conductance varies with the gate voltage on the scale which is parametrically small compared with the width of the mixed-valence region.

We thank N. Andrei, V. Cheianov, V.I. Falko, L.I. Glazman, A.J. Millis, and S. Tarucha for valuable discussions. This work was supported by the Nanoscience/ Nanoengineering Research Program of Georgia Tech, by EC RTN2-2001-00440 "Spintronics", Project OTKA D048665, and by the János Bolyai Scholarship.

[1] L.P. Kouwenhoven et al., in Mesoscopic Electron Transport, eds. L.L. Sohn et al. (Kluwer, Dordrecht, 1997), p. 105.

[2] M. Pustilnik and L.I. Glazman, J. Phys. Condens. Matter 16, R513 (2004); L.I. Glazman and M. Pustilnik, 
cond-mat/0501007

[3] W.G. van der Wiel et al., Science 289, 2105 (2000); Y. Ji, M. Heiblum and H. Shtrikman, Phys. Rev. Lett. 88 076601 (2002).

[4] A dot with even $N$ will have $S=1$ if the spacing between the two single-particle levels closest to the Fermi level is very small [2, 5, 6, 7]. This condition is easy to satisfy since the spacing is tunable [8]. However, having $S>1$ (e.g., $S=3 / 2$ for odd $N$ ) requires three or more levels to be nearly degenerate, which happens rarely, if ever.

[5] R.M. Potok et al., Phys. Rev. Lett. 91, 016802 (2003); J.A. Folk et al., Phys. Scripta T90, 26 (2001); S. Lindemann et al., Phys. Rev. B 66, 195314 (2002).

[6] P.W. Brouwer, Y. Oreg, and B.I. Halperin, Phys. Rev. B 60, R13977 (1999); H.U. Baranger, D. Ullmo, and L. I. Glazman, Phys. Rev. B 61, R2425 (2000).

[7] M. Pustilnik and L.I. Glazman, Phys. Rev. Lett. 87, 216601 (2001).

[8] W.G. van der Wiel et al., Phys. Rev. Lett. 88, 126803 (2002); D.M. Zumbühl et al., Phys. Rev. Lett. 93, 256801
(2004).

[9] Note that in Eq. (3) the phase shifts are defined $\bmod \pi$ (i.e. $\delta_{s}$ and $\delta_{s}+\pi$ are equivalent). The ambiguity is removed in Eq. (8) by setting $\delta_{s}=0$ for $N_{0} \rightarrow-\infty$.

[10] P.W. Anderson, Journ. of Phys. C 3, 2436 (1970).

[11] P. Nozières, J. Low Temp. Phys. 17, 31 (1974); P. Nozières and A. Blandin, J. Phys. (France) 41, 193 (1980).

[12] N. Andrei, K. Furuya and J.H. Lowenstein, Rev. Mod. Phys. 55, 331 (1983); A.M. Tsvelick and P.B. Wiegmann, Adv. Phys. 32, 453 (1983).

[13] K.G. Wilson, Rev. Mod. Phys. 47, 773 (1975); H.R. Krishna-murthy, J.W. Wilkins, and K.G. Wilson, Phys. Rev. B 21, 1003 (1980); Phys. Rev. B 21, 1044 (1980).

[14] W. Hofstetter and H. Schoeller, Phys. Rev. Lett. 88, 016803 (2001); M. Garst et al., Phys. Rev. B 69, 214413 (2004).

[15] M. Pustilnik, L.I. Glazman, and W. Hofstetter, Phys. Rev. B 68, 161303(R) (2003). 